ANNALES

POLONICI MATHEMATICI

$86.3(2005)$

\title{
On some elliptic boundary-value problems with discontinuous nonlinearities
}

\author{
by Giovanni Anello (Messina)
}

\begin{abstract}
We establish two existence results for elliptic boundary-value problems with discontinuous nonlinearities. One of them concerns implicit elliptic equations of the form $\psi(-\Delta u)=f(x, u)$. We emphasize that our assumptions permit the nonlinear term $f$ to be discontinuous with respect to the second variable at each point.
\end{abstract}

1. Introduction. Throughout, $\Omega$ is a nonempty open bounded set in $\mathbb{R}^{n}(n \geq 3)$ with smooth boundary $\partial \Omega, a$ is a real positive number, $p$ is a real number strictly greater than $n / 2$, and $f: \Omega \times \mathbb{R} \rightarrow \mathbb{R}, \psi:[a,+\infty[\rightarrow \mathbb{R}$ are given functions.

This paper is motivated by the results of [7] and [8] where some elliptic boundary-value problems with discontinuous nonlinearities are studied. Specifically, in these papers, the following two problems are considered:

$$
\begin{cases}-\Delta u(x)=f(x, u(x)) & \text { in } \Omega, \\ u=0 & \text { on } \partial \Omega,\end{cases}
$$

and

$$
\begin{cases}\psi(-\Delta u(x))=f(x, u(x)) & \text { in } \Omega, \\ u=0 & \text { on } \partial \Omega,\end{cases}
$$

where $\Delta$ is the Laplacian operator; for both, existence of strong solutions is established. As is well known, a strong solution for problem $(P)$ or $\left(P_{1}\right)$ is a function $u \in W^{2, p}(\Omega) \cap W_{0}^{1, p}(\Omega)$ satisfying the corresponding equation for almost all $x \in \Omega$. There is wide literature on the existence of solutions for problem $(P)$, and when $f$ is a Carathéodory function, variational methods are usually employed.

Here we are interested in the case where $f$ can be discontinuous with respect to the second variable. In this connection we refer to [7] and [8] (and the references therein). In particular, Theorems 3.1 and 4.2 of [7] give

2000 Mathematics Subject Classification: 35J25, 26E25.

Key words and phrases: elliptic equations, discontinuous nonlinearities, strong solutions, differential inclusions. 
the existence of a strong solution for problem $(P)$ and $\left(P_{1}\right)$ respectively, assuming that $f(x, \cdot)$ is a Riemann-measurable function for almost all $x \in \Omega$ and, in the case of problem $\left(P_{1}\right)$, that $f$ is also independent of $x \in \Omega$. We recall that a Riemann-measurable function is a function whose set of discontinuity points has Lebesgue measure 0 . In the same paper it is pointed out that the assumption on $f$ cannot be weakened. Indeed, in Remark 3.2 of [7] it is shown that if $f(x, \cdot)$ is almost everywhere equal to a Riemannmeasurable function for almost all $x \in \Omega$ only, then problem $(P)$ may not have any strong solution. On the other hand, Theorem 3.1 of [8] gives the existence of a strong solution for problem $\left(P_{1}\right)$ this time allowing $f$ to depend on $x \in \Omega$ and assuming hypotheses substantially different from those of Theorem 4.2 of [7].

The purpose of the present paper is twofold: we give versions of Theorems 3.1 and 4.2 of [7] in which $f(x, \cdot)$ is supposed to be almost everywhere equal to a Riemann-measurable function for almost all $x \in \Omega$ extending, at the same time, the second one to the nonautonomous case. In the latter case, our result and Theorem 3.1 of [8] will turn out to be mutually independent. To prove the existence result relating to problem $\left(P_{1}\right)$ we will use a recent selection theorem established in [1].

2. Basic definitions and notations. Let $X, Y$ be two nonempty sets. A multifunction $F$ from $X$ into $Y$ is a function from $X$ into the family of all subsets of $Y$ and we briefly denote it by $F: X \rightarrow 2^{Y}$. The set $\{(x, y) \in$ $X \times Y: y \in F(x)\}$ is called the graph of $F$. For each $A \subseteq Y$, we denote by $F^{-}(A)$ the set $\{x \in X: F(x) \cap A \neq \emptyset\}$. We say that a function $f: X \rightarrow Y$ is a selection of $F$ if $f(x) \in F(x)$ for all $x \in X$. If $X, Y$ are topological spaces, a multifunction $F: X \rightarrow 2^{Y}$ is called lower semicontinuous (briefly l.s.c.) at $x \in X$ if for any $y \in F(x)$ and any neighborhood $V$ of $y$ there exists a neighborhood $U$ of $x$ such that

$$
F(z) \cap V \neq \emptyset \quad \text { for all } z \in U .
$$

If $(X, \Im)$ is a measurable space and $Y$ is a topological space, a multifunction $F: X \rightarrow 2^{Y}$ is called measurable when $F^{-}(A) \in \Im$ for any open set $A \subseteq Y$.

We denote by $\mathcal{L}(\Omega)$ the Lebesgue $\sigma$-algebra of $\Omega$ and by $m_{n}$ the Lebesgue measure in $\mathbb{R}^{n}$. Also, the symbol $\mathcal{B}(\mathbb{R})$ stands for the Borel $\sigma$-algebra of $\mathbb{R}$. For a subset $A$ of $\mathbb{R}^{n}, \overline{\mathrm{co}}(A)$ and $\operatorname{int}(A)$ will denote the closed convex hull and the interior of $A$ respectively. If $d$ is the Euclidean distance in $\mathbb{R}^{n}$ and $A$ is a nonempty set in $\mathbb{R}^{n}$, we put $d(x, A)=\inf _{y \in A} d(x, y)$ for all $x \in \mathbb{R}^{n}$. Finally, we denote by $\|\cdot\|_{p}$ the usual norm in $L^{p}(\Omega)$.

To close this section, recall that, thanks to Theorem 2 of [11], one has

$$
\underset{x \in \Omega}{\operatorname{ess} \sup }|u(x)| \leq B\|\Delta u\|_{p} \quad \text { for every } u \in W^{2, p}(\Omega) \cap W_{0}^{1, p}(\Omega)
$$


where

$$
B=[m(\Omega)]^{2 / n-1 / p} \frac{\Gamma\left(1+\frac{n}{2}\right)}{\pi n(n-2)}\left[\frac{\Gamma\left(1+\frac{p}{p-1}\right) \Gamma\left(\frac{n}{n-2}\right)-\frac{p}{p-1}}{\Gamma\left(\frac{n}{n-2}\right)}\right]^{1-1 / p}
$$

and $\Gamma$ denotes the Gamma function.

3. Main results. In this section we state and prove the main results. Throughout, we briefly write a.a. for "almost all" .

Theorem 1. Let $\beta \in L^{p}(\Omega)$ with $\beta \neq 0$ and $A=\left[-B\|\beta\|_{p}, B\|\beta\|_{p}\right]$. Assume that there exist $E \subseteq A$ with $m_{1}(E)=0$ and a function $g: \Omega \times \mathbb{R} \rightarrow \mathbb{R}$ satisfying the following properties:

$\left(\alpha_{1}\right)\{t \in A: g(x, \cdot)$ is discontinuous at $t$ or $g(x, t) \neq f(x, t)\} \subseteq E$ for a.a. $x \in \Omega$;

$\left(\alpha_{2}\right) g(\cdot, t)$ is measurable for a.a. $t \in A$;

$\left(\alpha_{3}\right) \sup _{t \in A}|g(x, t)| \leq \beta(x)$ for a.a. $x \in \Omega$;

$\left(\alpha_{4}\right)$ there exists an open set $D \supseteq E$ such that

$$
\underset{x \in \Omega}{\operatorname{essinf}} \inf _{t \in D} g(x, t)>0 \quad \text { or } \quad \operatorname{ess} \sup _{x \in \Omega} \sup _{t \in D} g(x, t)<0 .
$$

Then there exists a strong solution $u$ of problem $(P)$ satisfying

$$
|\Delta u(x)| \leq \sup _{t \in A}|g(x, t)| \quad \text { for a.a. } x \in \Omega .
$$

Proof. Without loss of generality, we can suppose that conditions $\left(\alpha_{1}\right)$ and $\left(\alpha_{3}\right)$ hold for all $x \in \Omega$. For every $x \in \Omega$, we define

$$
\widehat{g}(x, t)= \begin{cases}g\left(x,-B\|\beta\|_{p}\right) & \text { if } t<-B\|\beta\|_{p}, \\ g(x, t) & \text { if }|t| \leq B\|\beta\|_{p}, \\ g\left(x, B\|\beta\|_{p}\right) & \text { if } t>B\|\beta\|_{p} .\end{cases}
$$

Clearly $\left(\alpha_{1}\right)$ holds with $\widehat{g}$ in place of $g$. Observe that by $\left(\alpha_{2}\right)$ we can find a countable set $P \subseteq \mathbb{R} \backslash E$ dense in $\mathbb{R}$ such that $\widehat{g}(\cdot, t)$ is measurable for all $t \in P$. Moreover, by $\left(\alpha_{3}\right)$, we can suppose that the function $\widehat{g}(x, \cdot)$ is bounded for all $x \in \Omega$. Thus, thanks to Proposition 2 of [3] (which is proved assuming that $\Omega$ is a real interval, but it is easy to see that the same holds if $\Omega$ is a bounded open subset of $\mathbb{R}^{n}$ ), the multifunction $F: \Omega \times \mathbb{R} \rightarrow 2^{\mathbb{R}}$ defined by

$$
F(x, t)=\bigcap_{m \in \mathbb{N}} \overline{\operatorname{co}}\left(\bigcup_{y \in P,|y-t| \leq 1 / m}\{\widehat{g}(x, y)\}\right)
$$

has the following properties:

(a) $F(x, t)$ is nonempty and convex for all $(x, t) \in \Omega \times \mathbb{R}$;

(b) $F(\cdot, t)$ is measurable for all $t \in \mathbb{R}$; 
(c) $F(x, \cdot)$ has closed graph for all $x \in \Omega$;

(d) if $x \in \Omega$ and $\widehat{g}(x, \cdot)$ is continuous at $t \in \mathbb{R}$, then $F(x, t)=\{\widehat{g}(x, t)\}$. Now, thanks to Proposition 1 of [9] it is easy to deduce that

$$
\sup _{t \in A} d(0, F(x, t))=\sup _{t \in A \cap P} d(0, F(x, t))
$$

for all $x \in \Omega$ (see, for instance, the proof of Theorem 3.1 of [7]). Consequently, the function $x \in \Omega \mapsto \sup _{t \in A} d(0, F(x, t))$ is measurable. Moreover, from condition $\left(\alpha_{3}\right)$, this function belongs to $L^{p}(\Omega)$ and

$$
\left\|\sup _{t \in A} d(0, F(\cdot, t))\right\|_{L^{p}(\Omega)} \leq\|\beta\|_{p} .
$$

At this point, we can apply Theorem 2.2 of [7]. Hence, there exists $u \in$ $W^{2, p}(\Omega) \cap W_{0}^{1, p}(\Omega)$ such that

$$
-\Delta u(x) \in F(x, u(x))
$$

and

$$
|\Delta u(x)| \leq \sup _{t \in A} d(0, F(x, t))
$$

for a.a. $x \in \Omega$. Observe that from (3) and (4) and in view of (1) we have

$$
\underset{x \in \Omega}{\operatorname{ess} \sup _{x}}|u(x)| \leq B\|\Delta u(x)\|_{L^{p}(\Omega)} \leq B\left\|\sup _{t \in A} d(0, F(x, t))\right\|_{L^{p}(\Omega)} \leq B\|\beta\|_{p},
$$
that is,

$$
u(x) \in A \quad \text { for a.a. } x \in \Omega .
$$

Now, put

$$
\Omega_{0}=\{x \in \Omega: u(x) \in E\} .
$$

We claim that $m_{n}\left(\Omega_{0}\right)=0$. Indeed, by Proposition 2.1 of [7] one has

$$
\Delta u(x)=0 \in F(x, u(x))
$$

for almost all $x \in \Omega_{0}$. On the other hand, by condition $\left(\alpha_{4}\right)$, we have

$$
\underset{x \in \Omega}{\operatorname{ess} \inf } \inf _{t \in D} \inf F(x, t) \geq \underset{x \in \Omega}{\operatorname{essinf}} \inf _{t \in D} g(x, t)>0
$$

or

$$
\underset{x \in \Omega}{\operatorname{ess} \sup } \sup _{t \in D} \sup F(x, t) \leq \operatorname{ess}_{x \in \Omega} \sup _{t \in D} g(x, t)<0 .
$$

Clearly, (6) together with (7) or (8) imply $m_{n}\left(\Omega_{0}\right)=0$. This latter fact, the definition of $\widehat{g}$, condition $\left(\alpha_{1}\right)$, properties (d) and (3)-(5) imply

$$
-\Delta u(x)=f(x, u(x))
$$

and

$$
|\Delta u(x)| \leq \sup _{t \in A}|g(x, t)|
$$

for a.a. $x \in \Omega$. This completes the proof. 
REMARK 1. As observed in the introduction, an example in Remark 3.2 of [7] shows that if $f$ is almost everywhere equal to a function fulfilling all the assumptions of Theorem 3.1 of [7], then problem $(P)$ may not have any strong solution. Precisely, for $f$ defined by

$$
f(x, t)= \begin{cases}1 & \text { if }(x, t) \in \Omega \times \mathbb{Q}, \\ 0 & \text { otherwise }\end{cases}
$$

problem $(P)$ cannot have any strong solution. Observe that this $f$ does not satisfy the hypotheses of Theorem 1 . Indeed, it is easy to note that there is no function $g$ fulfilling both $\left(\alpha_{1}\right)$ and $\left(\alpha_{4}\right)$. On the other hand, if we define

$$
f(x, t)= \begin{cases}0 & \text { if }(x, t) \in \Omega \times \mathbb{Q}, \\ 1 & \text { otherwise }\end{cases}
$$

then for $g(x, t)=1$ for all $(x, t) \in \Omega \times \mathbb{R}$ all the hypotheses of Theorem 1 are satisfied, and so problem $(P)$ admits a strong solution. We emphasize that in this latter case $f$ is discontinuous at each point of $\mathbb{R}$.

REMARK 2. We observe that condition $\left(\alpha_{3}\right)$ of Theorem 1 is weaker than condition $\left(\alpha_{3}\right)$ of Theorem 3.1 of [7]. Indeed, the measurability of the function $x \in \Omega \mapsto \sup _{t \in A}|g(x, t)|$ is not required.

To prove the next result we need the following selection theorem for multifunctions of two variables:

Theorem A (Theorem 2 of [1]). Let T, $X$ be Polish spaces and let $\mu, \psi$ be positive regular Borel measures on $T$ and $X$, respectively, with $\mu$ finite and $\psi \sigma$-finite. Let $S$ be a separable metric space, $F: T \times X \rightarrow 2^{S}$ a multifunction with nonempty complete values, and let $E \subseteq X$ be a given set. Finally, let $\mathcal{B}(X)$ be the Borel $\sigma$-algebra of $X$ and $\mathcal{T}_{\mu}$ the completion of the Borel $\sigma$-algebra of $T$ with respect to $\mu$. Assume that:

(i) $F$ is $\mathcal{T}_{\mu} \otimes \mathcal{B}(X)$-measurable;

(ii) $\{x \in X: F(t, \cdot)$ is not lower semicontinuous at $x\} \subseteq E$ for a.a. $t \in T$.

Then there exists a selection $\phi: T \times X \rightarrow S$ of $F$ and a negligible set $R \subseteq X$ such that:

(i) $)^{\prime} \phi(\cdot, x)$ is $\mathcal{T}_{\mu}$-measurable for each $x \in X \backslash(E \cup R)$;

(ii) $)^{\prime}\{x \in X: \phi(t, \cdot)$ is not continuous at $x\} \subseteq E \cup R$ for a.a. $t \in T$.

Now, we are able to prove our second main result. From now on, if $C \subseteq \mathbb{R}$, $\mathcal{B}(C)$ will denote the Borel $\sigma$-algebra of $C$.

Theorem 2. Let $\beta \in L^{p}(\Omega)$ with $\beta \neq 0$ and put $A=\left[-B\|\beta\|_{p}, B\|\beta\|_{p}\right]$. Assume that there exist $E, E_{1} \subset A$ with $m_{1}\left(E \cup E_{1}\right)=0$ and $E_{1}$ closed, and a function $g: \Omega \times \mathbb{R} \rightarrow \mathbb{R}$ satisfying the following properties: 
$\left(\beta_{1}\right)\{t \in A: g(x, \cdot)$ is discontinuous at $t\} \subseteq E_{1}$ and $\{t \in A: g(x, t) \neq f(x, t)\} \subseteq E$ for a. $a . x \in \Omega$;

$\left(\beta_{2}\right) \psi$ is continuous in $\left[a,+\infty\left[\right.\right.$ and $\psi^{-1}(\sigma)$ has empty interior for every $\sigma \in \operatorname{int} \psi([a,+\infty])$;

$\left(\beta_{3}\right) g(\cdot, t)$ is measurable for a.a. $t \in A$;

$\left(\beta_{4}\right)$ if one puts

$$
v(x)=\underset{t \in A}{\operatorname{essinf}} g(x, t) \quad \text { and } \quad z(x)=\operatorname{esssup}_{t \in A} g(x, t)
$$

for all $x \in \Omega$, then $[v(x), z(x)] \subseteq \psi\left(\left[a,+\infty[)\right.\right.$ and $\psi^{-1}([v(x), z(x)])$ $\subseteq[a, \beta(x)]$ for a.a. $x \in \Omega$.

Then there exists a strong positive solution of problem $\left(P_{1}\right)$.

Proof. Without loss of generality, we can suppose that the conditions $\left(\beta_{1}\right)$ and $\left(\beta_{4}\right)$ hold for all $x \in \Omega$. By $\left(\beta_{2}\right)$ we can find a countable set $P \subseteq A \backslash E_{1}$ dense in $A$ such that $g(\cdot, t)$ is measurable for all $t \in P$. Moreover, it is easy to see that

$$
v(x)=\inf _{t \in A \backslash E_{1}} g(x, t) \quad \text { and } \quad z(x)=\sup _{t \in A \backslash E_{1}} g(x, t)
$$

for all $x \in \Omega$. Hence, $g_{\mid \Omega \times\left(A \backslash E_{1}\right)}$ is $\mathcal{L}(\Omega) \otimes \mathcal{B}\left(A \backslash E_{1}\right)$-measurable by the Lemma on p. 198 of [6]. So, by Lemma III.39 of [2], the functions $v$ and $z$ are measurable. Now, define

$$
\phi(x, t)= \begin{cases}g(x, t) & \text { if }(x, t) \in \Omega \times\left(A \backslash E_{1}\right), \\ z(x) & \text { if }(x, t) \in \Omega \times E_{1} .\end{cases}
$$

Since $E_{1}$ is closed, condition $\left(\beta_{1}\right)$ implies that

$$
\{t \in A: \phi(x, \cdot) \text { is discontinuous at } t\} \subseteq E_{1} .
$$

Moreover, $\phi$ turns out to be $\mathcal{L}(\Omega) \otimes \mathcal{B}(A)$-measurable and satisfies

$$
v(x) \leq \phi(x, t) \leq z(x)
$$

for all $(x, t) \in \Omega \times A$. At this point, observe that the function $\psi$ fulfils all the hypotheses of Theorem 2.4 of [10]. Hence, there exists a set $Y \subseteq[a,+\infty[$ such that $\psi^{-1}(\sigma) \cap Y$ is nonempty and closed in $\mathbb{R}$ for each $\sigma \in \psi([a,+\infty[)$ and the multifunction $\psi^{-1}(\cdot) \cap Y$ is l.s.c. in $\psi([a,+\infty[)$. Now, put

$$
\Gamma(x, t)= \begin{cases}\psi^{-1}(\phi(x, t)) \cap Y & \text { if }(x, t) \in \Omega \times A, \\ \mathbb{R} & \text { otherwise. }\end{cases}
$$

Then $\Gamma$ is an $\mathcal{L}(\Omega) \otimes \mathcal{B}(\mathbb{R})$-measurable multifunction and, further, by (9) one has

$$
\{t \in \mathbb{R}: \Gamma(x, \cdot) \text { is not l.s.c. at } t\} \subseteq E_{1} .
$$


Consequently, by Theorem A, there exist $R \subseteq \mathbb{R}$ with $m_{1}(R)=0$ and a selection $\gamma$ of $\Gamma$ such that

$$
\{t \in \mathbb{R}: \gamma(x, \cdot) \text { is discontinuous at } t\} \subseteq E_{1} \cup R
$$

for a.a. $x \in \Omega$ and $\gamma(\cdot, t)$ measurable for all $t \in \mathbb{R} \backslash\left(E_{1} \cup R\right)$. From this latter property we can find a countable set $P_{1} \subseteq \mathbb{R} \backslash\left(E_{1} \cup R\right)$, dense in $\mathbb{R}$, such that $\gamma(\cdot, t)$ is measurable for all $t \in P_{1}$. Also, by conditions $\left(\beta_{4}\right),(10)$ and since

$$
\gamma(x, t) \in \psi^{-1}(\phi(x, t)) \quad \text { for all }(x, t) \in \Omega \times A
$$

we deduce that

$$
\begin{aligned}
a \leq \gamma(x, t) & \leq \sup _{t \in A} \sup \psi^{-1}(\phi(x, t)) \\
& \leq \sup \psi^{-1}([v(x), z(x)]) \leq \beta(x)
\end{aligned}
$$

for all $(x, t) \in \Omega \times A$. At this point, we can apply Theorem 1 . Hence, there exists $u \in W^{2, p}(\Omega) \cap W_{0}^{1, p}(\Omega)$ such that

$$
-\Delta u(x)=\gamma(x, u(x))
$$

and

$$
|\Delta u(x)| \leq \sup _{t \in A} \gamma(x, t) \leq \beta(x)
$$

for a.a. $x \in \Omega$. In particular, by (16) and (1), one has $\operatorname{ess} \sup _{x \in \Omega}|u(x)| \leq$ $B\|\beta\|_{p}$, that is,

$$
u(x) \in A \quad \text { for a.a. } x \in \Omega .
$$

Taking into account (14) and (15), we can argue as in the proof of Theorem 1 to deduce that

$$
m_{n}\left(\left\{x \in \Omega: u(x) \in E \cup E_{1}\right\}\right)=0 .
$$

Consequently, by the definition of $\phi$ and conditions $\left(\beta_{1}\right),(13),(15),(17)$ and (18) one has

$$
\psi(-\Delta u(x))=f(x, u(x))
$$

for a.a. $x \in \Omega$. Moreover, the Maximum Principle and (14), (15) and (17) imply that $u$ is positive. This completes the proof.

REMARK 3. Notice that Theorem 2 is a nonautonomous version of Theorem 4.2 of [7].

REMARK 4. When the function $g$ of Theorem 2 does not depend on $x \in \Omega$, then we can take the function $\beta$ equal to a constant $\varrho$. So we have $A=\left[-B m_{n}(\Omega)^{1 / p} \varrho, B m_{n}(\Omega)^{1 / p} \varrho\right]$ and condition $\left(\beta_{4}\right)$ becomes

$$
\begin{aligned}
\left(\beta_{4}^{\prime}\right) & g\left(\left[-B m_{n}(\Omega)^{1 / p} \varrho, B m_{n}(\Omega)^{1 / p} \varrho\right]\right) \subseteq \psi([a,+\infty]) \text { and } \\
& \psi^{-1}\left(g\left(\left[-B m_{n}(\Omega)^{1 / p} \varrho, B m_{n}(\Omega)^{1 / p} \varrho\right]\right)\right) \subseteq[a, \varrho] .
\end{aligned}
$$


We observe that condition $\left(\beta_{4}^{\prime}\right)$ is stronger than condition $\left(c_{3}\right)$ of Theorem 4.2 of [7]. Hence, the former does not generalize the latter to the nonautonomous case. Nevertheless, we point out that condition $\left(c_{3}\right)$ of Theorem 4.2 of [7] must be replaced with $\left(\beta_{4}^{\prime}\right)$, otherwise inequality (14) and the subsequent inclusion in the proof of that result may not be true, as is easily checked.

REMARK 5. We observe that Theorem 3.1 of [8] deals with the vectorial case of problem $\left(P_{1}\right)$, namely $\mathbb{R}$ is replaced by $\mathbb{R}^{h}$ where $h$ is an integer greater than or equal to 1 . When $h=1$, it is immediate to check that this result and Theorem 2 are mutually independent.

\section{References}

[1] G. Anello and P. Cubiotti, Parametrization of Riemann-measurable selections for multifunctions of two variables with application to differential inclusions, Ann. Polon. Math. 83 (2004), 179-187.

[2] C. Castaing and M. Valadier, Convex Analysis and Measurable Multifunctions, Springer, Berlin, 1977.

[3] P. Cubiotti, Non-autonomous vector integral equations with discontinuous righthand side, Comm. Math. Univ. Carolin. 38 (1997), 241-246.

[4] E. Hewitt and K. Stromberg, Real and Abstract Analysis, Springer, Berlin, 1965.

[5] E. Klein and A. C. Thompson, Theory of Correspondences, Wiley, New York, 1984.

[6] A. Kucia, Scorza Dragoni type theorems, Fund. Math. 138 (1991), 197-203.

[7] A. S. Marano, Elliptic boundary-value problem with discontinuous nonlinearities, Set-Valued Anal. 3 (1995), 167-180.

[8] -, Implicit elliptic boundary-value problem with discontinuous nonlinearities, ibid. 4 (1996), 287-300.

[9] O. Naselli Ricceri and B. Ricceri, An existence theorem for inclusions of the type $\Psi(u)(t) \in F(t, \Phi(u)(t))$ and application to a multivalued boundary problem, Appl. Anal. 38 (1990), 259-270.

[10] B. Ricceri, Sur la semi-continuité inférieure de certaines multifonctions, C. R. Acad. Sci. Paris 294 (1982), 265-267.

[11] G. Talenti, Elliptic equations and rearrangements, Ann. Scuola Norm. Sup. Pisa Cl. Sci. (4) 3 (1976), 697-718.

Department of Mathematics

University of Messina

98166 S.Agata, Messina, Italy

E-mail: anello@dipmat.unime.it 